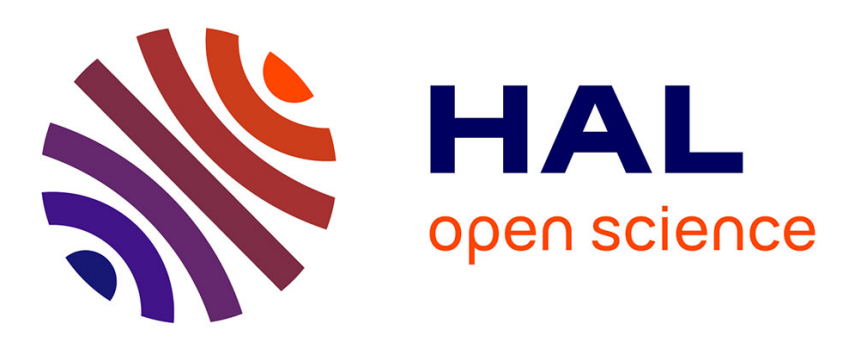

\title{
Logique de l'emploi et stratégies des dirigeants territoriaux dans un nouveau contexte institutionnel
}

\author{
Laurence Durat
}

\section{To cite this version:}

Laurence Durat. Logique de l'emploi et stratégies des dirigeants territoriaux dans un nouveau contexte institutionnel. Revue française d'administration publique, 2012, 141 (1), pp.223-237. 10.3917/rfap.141.0223 . hal-03096372

\section{HAL Id: hal-03096372 \\ https://hal.science/hal-03096372}

Submitted on 4 Jan 2021

HAL is a multi-disciplinary open access archive for the deposit and dissemination of scientific research documents, whether they are published or not. The documents may come from teaching and research institutions in France or abroad, or from public or private research centers.
L'archive ouverte pluridisciplinaire HAL, est destinée au dépôt et à la diffusion de documents scientifiques de niveau recherche, publiés ou non, émanant des établissements d'enseignement et de recherche français ou étrangers, des laboratoires publics ou privés. 


\title{
Logique de l'emploi et stratégies des dirigeants territoriaux dans un nouveau contexte institutionnel
}

\author{
Laurence Durat \\ Maître de Conférences \\ Université de Haute-Alsace \\ LISEC EA 2310 Strasbourg
}

Résumé :

La politisation accrue de l'action publique locale et l'emploi fonctionnel introduit par la loi de 1987 relativisent la notion de garantie de l'emploi et transforment les parcours des hauts fonctionnaires territoriaux. Rapidement la logique de l'emploi a surplombé celle de la carrière pourtant très ancrée dans la fonction publique française. Les liens d'interdépendance entre élus et directeur général des services sont redéfinis et cette modification institutionnelle donne le jour à un nouveau mode d'exercice du métier de DGS. Confrontés à une plus grande instabilité de leur fonction depuis 15 ans, les dirigeants de l'administration territoriale développent des stratégies adaptatives.

Mots-clés : dirigeants territoriaux - carrières - emploi fonctionnel 
En France, comme dans la majorité des Etats membres de l'Union européenne, la décentralisation des pouvoirs s'est traduite à partir des lois de 1982-83 par des transferts de responsabilité et de moyens du gouvernement central aux organisations infra-nationales. Cette dynamique de décentralisation approfondie par de nouveaux transferts de compétences (Acte II de la décentralisation) a renforcé l'autonomie locale et régionale des collectivités territoriales au plan de la décision et de l'action. Pour mettre en œuvre les services publics locaux relevant de leurs compétences, les collectivités infra-nationales ont renforcé progressivement leurs capacités administratives locales. Animées par des exécutifs élus au suffrage universel, les collectivités territoriales ont fait face à la complexité croissante de l'action publique par la professionnalisation de leurs élites administratives. Le poids du secteur public territorial dans l'intervention publique n'a cessé de croître, le portant à près de $55 \%$ des dépenses publiques en France ${ }^{1}$ en 2005 . En outre, l'autonomie locale s'accompagne du contrôle a posteriori sur les actes administratifs, budgétaires ou financiers.

Les responsabilités qui incombent aux directeurs généraux des services sont donc importantes, au plan juridique et financier comme au plan de la mise en ouvre de l'action publique territoriale et elles se doublent de particularités liées au contexte de leur activité. En effet, ils ont à prendre en compte l'impératif politique (ils agissent pour le compte des élus qui les recrutent) ainsi que la temporalité de l'action (la durée du mandat électif), ils doivent s'adapter aux territoires différents qu'ils ont à connaître (au plan socio-économique, politique et historique) et sont eux-mêmes soumis à une mobilité à répétition, en fonction des résultats des élections ; le 'spoil system', dans lequel les élites administratives accompagnent les élites politiques dans leur ascension mais aussi dans leur chute se diffuse progressivement ( Le Saout , 2009² ; Perrin, 2009) et plus que la réforme territoriale ne l'anticipait.

Ces conditions nouvelles d'exercice de leur activité ont des conséquences directes sur les modes d'intervention des dirigeants, sur l'identité professionnelle qu'elles génèrent et sur les équilibres personnels qui sont recherchés. Ni comparables à celles des cadres supérieurs des entreprises privées, même si elles s'en rapprochent partiellement, ni assimilables à celles de leurs collègues de la fonction publique d'Etat, les carrières des dirigeants territoriaux et les

\footnotetext{
${ }^{1}$ Les collectivités territoriales dans l'union européenne, Dexia Editions, 2008.

${ }^{2}$ Le Saout Rémy (2009). "L'intercommunalité et mutation des liens entre les directeurs des services municipaux et les maires ", Revue Française d'Administration Publique, n 128 ; Bernard Perrin, (2009) « Les aléas de la diversité dans la fonction publique territoriale », Revue française d'administration publique, ${ }^{\circ} 132$.
} 
stratégies qui en découlent présentent une relation d'emploi tout à fait originale et en pleine évolution.

Pour mener à bien cette analyse, nous nous appuierons sur les divers travaux sur les dirigeants territoriaux que nous avons menés depuis 6 ans, dont principalement deux sources majeures : une étude comparée sur le statut, les compétences et les responsabilités des dirigeants des administrations locales et régionales en Europe menée par l'Union des Dirigeants Territoriaux Européens (UDITE) et une étude sur l'activité des dirigeants territoriaux menée dans le cadre d'un programme de recherches sur des dirigeants de la sphère publique (dirigeants hospitaliers, territoriaux, du travail, etc.) à la demande des écoles d'application formant ces dirigeants, programme coordonné par le Conservatoire National des Arts et Métiers (CNAM) et le réseau des écoles de service public (RESP) dont les détails figurent en annexe méthodologique en fin de chapitre.

\section{Un contexte institutionnel en constante mutation}

Dans la fonction publique territoriale (FPT) plus encore que dans la fonction publique d'Etat, la recomposition entre secteur public et société civile a été profonde et relativement rapide. L'appareil administratif s'est avéré relativement flexible, absorbant la décentralisation, l'Europe, la modernisation des services publics et la transformation de modes de gestion. Progressivement, les hauts fonctionnaires sont appelés à se transformer en managers et le statut social de ces cadres supérieurs évolue pour accompagner ce changement.

\section{Un système des carrières en voie d'affaiblissement}

A la diversité des conditions d'emploi offertes d'une collectivité à l'autre s'est substitué depuis environ 25 ans (loi du 26 janvier 1984) un statut général unique regroupant le personnel des communes, des départements, des régions et de leurs établissements publics à caractère administratif. Il a la particularité de tenter de concilier l'inconciliable, à savoir l'autonomie de la collectivité locale et la garantie de conditions d'emploi similaires à celles des agents publics. Si cette parité est globalement atteinte pour les catégories B (encadrement intermédiaire) et $\mathrm{C}$ (fonctions d'exécution), il en va autrement des catégories $\mathrm{A}$ (fonctions de conception et de direction) dont font partie les dirigeants. Les collectivités disposant de la pleine compétence pour créer les postes, nommer, décider de l'avancement et licencier les agents locaux, ne se privent pas d'exercer cette autonomie pour les postes les plus sensibles.

A la tête des collectivités territoriales (communes, départements, régions et établissements publics de coopération intercommunale), les fonctionnaires de haut niveau (cadre d'emplois 
de catégorie A de la fonction publique) sont recrutés après avoir obtenu un concours externe ou interne (accessible sous conditions de diplôme, le plus souvent de droit public ou de sciences politiques) de type administrateur (ou aussi Ingénieur scientifique, technique ou attaché). A cette formation initiale s'ajoute une formation dans une école d'application, l'INET (Institut National des Etudes Territoriales de Strasbourg), au sein de laquelle les élèves ont la qualité de fonctionnaires stagiaires.

La fonction publique territoriale française est régie par le système de la carrière, avec une séparation entre grade et emploi, ainsi le fonctionnaire est titulaire de son grade en fonction de son ancienneté et de son mérite (avancement d'échelon dans le grade) à l'intérieur d'un cadre d'emplois auquel il accède par concours ou promotion. Cela revient à dire qu'en cas de suppression du poste, l'agent n'est pas licencié mais réaffecté à un autre emploi. La carrière $\mathrm{du}$ fonctionnaire territorial relève de la collectivité ou du centre départemental de gestion. Mais ce système protecteur est notablement affecté par deux innovations concernant l'élite administrative : le recrutement direct et l'emploi fonctionnel.

L'encadrement supérieur est évalué par l'Observatoire du CNFPT ${ }^{3}$ à environ 2500 personnes, soit $0,7 \%$ de l'effectif total, dans une proportion qui varie selon les collectivités territoriales de $0,5 \%$ dans les communes à $3 \%$ dans les régions, dont $30 \%$ sont des emplois fonctionnels dans les agglomérations, départements et régions. Les personnes en poste sur des emplois fonctionnels sont majoritairement issus de la FPT (80\% dans les communes, 65\% dans les départements, $47 \%$ dans les régions) mais une autre voie de recrutement dérogatoire existe, (article 47 de la loi de 1984) celle du recrutement direct d'agents non titulaires à la condition de détenir un diplôme de niveau Bac+5 ou équivalent ou d'un niveau d'expérience professionnelle de 5 ans dans une administration ou un établissement public. La logique de carrière est progressivement effacée par la logique de l'emploi, comme l'observe Perrin (2005, p.585) : "on remarquera qu'on ne se trouve plus du tout dans un système de carrière et que ce mode de recrutement ou de nomination nous rapproche des dispositions en vigueur dans les pays anglo-saxons, notamment au Royaume-Uni où les personnels d'encadrement supérieur (chief officers) sont recrutés sur le seul fondement du diplôme ou des acquis professionnels équivalents et d'un entretien ${ }^{4}$.

\footnotetext{
${ }^{3}$ Centre National de la Fonction Publique Territoriale

${ }^{4}$ Bernard Perrin, «Les emplois de direction dans les collectivités territoriales : la capacité d'adaptation, vertu première », Revue française d'administration publique, n¹16, 2005, p. 583-594.
} 


\section{Une révolution silencieuse des carrières depuis 15 ans}

Une autre innovation bien plus agissante tend à individualiser les parcours de l'élite administrative: l'article 53 de la loi de 1984 concernant la nomination des directeurs généraux et de leurs adjoints des collectivités de plus de 3500 habitants sur 'emploi fonctionnel'. Une distinction s'opère ainsi entre les carrières statutaires et les emplois fonctionnels ou responsabilités et instabilité sont plus grandes. La traditionnelle disjonction des sphères administrative et politique «les élus passent, les fonctionnaires restent »n'est plus observée au sommet de l'organisation. Si le directeur général des services (DGS) et ses adjoints (DGA), sont nommés par le chef de l'exécutif local sur emploi fonctionnel à sa discrétion, ils sont également révocables par lui. Cette décision (un simple arrêté) n'a pas à être motivée, la décharge de fonction pouvant intervenir à tout moment, dans les faits on l'a observé surtout après renouvellement des élus d'une collectivité territoriale, mais pas seulement pour des raisons politiques. Depuis les élections municipales de 1995 (remplacement de quelques directeurs de services), le mouvement s'est largement amplifié lors des élections cantonales et régionales de 2004, puis la 'fin de détachement sur emploi fonctionnel' s'est systématisée, doublant l'alternance politique d'une alternance administrative d'équipes entières. Les conditions de fin d'emploi se sont donc transformées assez radicalement pour les dirigeants de la fonction publique territoriale.

Le DGS va donc intervenir dans un contexte où la stabilité n'est plus de mise : l'état-major administratif est en place pour quelques années puis révoqué et en situation de se retrouver un autre poste. Ce nouveau contexte d'exercice, signe de politisation accrue, calant la durée de l'activité du dirigeant sur le mandat électoral de l'élu, est à présent identifiée par les principaux intéressés comme faisant partie intégrante de leur carrière et acceptée au nom du libre choix par les exécutifs locaux de leurs collaborateurs. Une des conséquences en est la mobilité devenue un facteur essentiel dans le déroulement de la carrière des dirigeants, mobilité professionnelle comme géographique, qui ont à composer avec cette nouvelle dimension de leur activité.

\section{Modification des relations et des pratiques managériales}

Ainsi, en l'espace de 15 ans, les perspectives de carrière des élites territoriales ont totalement changé ; sur le versant positif, les parcours présentent l'avantage d'être plus diversifiés, de donner à connaître des collectivités ou établissements multiples, des niveaux d'intervention des politiques publiques différents, une plus grande responsabilisation des tâches et une participation intensive aux destinées des organisations infra-nationales, de même qu'une plus 
grande attractivité des rémunérations. Si les fonctionnaires territoriaux ont en commun avec les fonctionnaires d'Etat la grille indiciaire, la valeur de l'indice, ainsi que les modalités de calcul du traitement de base, de l'indemnité de résidence et du supplément familial de traitement, en revanche les primes et indemnités prévues par les textes sont instituées par chaque collectivité et engendrent, particulièrement au niveau des emplois fonctionnels, une grande disparité. Corrélativement à l'individualisation des parcours, les rémunérations sont elles aussi ajustées, et les recrutements de cadres extérieurs à la collectivité sur emplois fonctionnels sont d'autant plus attractifs que la part variable du salaire est importante. Les cadres dirigeants subissant une mise sous tension croissante, en écho à la pression des élus à la tête des collectivités territoriales, ces contreparties financières à leur grande disponibilité sont désormais attendues. Sur le plan matériel, les dirigeants sont donc généralement bien traités et ont davantage de compensations financières (logement et véhicule de fonction, frais de représentation). Cela va de pair avec la généralisation de la pratique de révocation des membres des équipes de direction, le parallèle avec le fonctionnement de l'entreprise est de plus en plus net, important dans le champ territorial une logique de contrat et des modes opératoires propres au management du modèle du marché -instabilité, évaluation de la performance, distanciation vis-à-vis de l'organisation, responsabilité individuelle de la formation, etc. (Vercher \& al. $2006^{5}$ ).

Mais nous faisons le choix dans cet article de montrer également le versant sombre de ces changements institutionnels, dans la mesure où l'envers de la médaille est peu abordé par la littérature et sous-estimé par les dirigeants territoriaux eux-mêmes comme par les autres partenaires de leur activité. Nous pensons cet objet d'étude digne d'intérêt et susceptible d'éclairer les pratiques politico-administratives locales. Leurs conséquences sont une transformation de la conception de leur rôle ainsi que des carrières en dents de scie, avec reculs, ralentissements, blocages, dans lesquelles compétences et disponibilité ne garantissent pas une progression linéaire et où les aléas politiques impactent directement les parcours des dirigeants territoriaux.

\section{Un rapport au politique et aux services transformé}

La prégnance et l'instabilité du rapport au politique dans l'exercice des fonctions de direction territoriale est de fait une donnée majeure à la fois dans la mise en œuvre même des actions

\footnotetext{
${ }^{5}$ Vercher C., Palpacuer F., Seignour A. XVIIe Congrès de l'AGRH - Le travail au coeur de la GRH, IAE de Lille et Reims Management School, novembre, Reims. (2006)
} 
publiques et dans son rejaillissement sur les dirigeants: la question de la compétence relationnelle spécifique qui est requise par la collaboration avec les élus et de la capacité à résister à la pression que cette proximité suscite est un critère discriminant tout autant que le professionnalisme des dirigeants en matière technique (économique, juridique, financière, etc.). Ces éléments nous amènent à aborder la relation entre le DGS et le chef de l'exécutif. De plus en plus ce dernier souhaite recruter dès sa prise de fonction un homme à sa convenance, préférant rompre la relation d'emploi au directeur attaché à la précédente mandature. Cette relation est fondatrice de toute activité du DGS. Dans les propos de tous les DGS, la qualité de la relation avec l'élu est citée comme déterminante de leur marge de manœuvre managériale : compréhension mutuelle, connivence, confiance sont les mots-clefs pour qualifier les interactions entre les deux personnes. "C'est à l'élu qu'appartient le pouvoir final de décision, la légitimité politique. Le relationnel avec l'élu, la traduction du message politique en démarche d'entreprise par le DGS est primordiale. C'est un prérequis à la fonction : la confiance pour avoir de l'autonomie, de la marge de mancuvre. » DGS Département. Le chef de l'exécutif (Maire ou Président) est celui qui recrute et évalue en $1^{\text {ère }}$ ligne le DGS, sa confiance est la seule garantie de fonctionnement du DGS, compte tenu de l'instabilité de la fonction de celui-ci : «Dès que la confiance est en doute, la direction générale est au ralenti, c'est très difficile à vivre, on a l'impression de ne pas compter du tout. » DGA Département. Cette relation nécessaire au fonctionnement de l'équipe est fondée sur l'entente entre le $1^{\mathrm{er}}$ élu et le $1^{\mathrm{er}}$ fonctionnaire de la collectivité : «il faut découvrir très rapidement quelles sont ses attentes ; alors ici moi j'ai de la chance d'avoir quelqu'un qui exprime au fur et à mesure, il y a une complicité, il faut trouver une complicité je pense, ça fait partie de la réussite(...). » DGS grande agglomération.

Les directeurs font état de certains moments ou quels que soient les réalisations et le management du DGS, l'insécurité de l'emploi affleure : «Pendant les périodes pré et post électorales le DG perd de l'influence, on tire à boulets rouges sur la technocratie. On est en train de perdre la capacité d'avoir une action commune. Le DG soit sera viré, soit il y aura un temps d'observation, d'adaptation. C'est clair que le DG est l'empêcheur de tourner en rond d'une structure. Tous les 3 ans, c'est cyclique, il y a un affaiblissement de la direction générale par les changements d'orientations, de personnes, de politiques. » DGA Département.

La temporalité politique produit donc simultanément une succession de mobilités des dirigeants de haut niveau et une relation ambivalente, à la fois impérative et fragile à l'autorité 
territoriale. En revanche, si une accélération de la mobilité des équipes de direction est constatée, il n'en est pas de même pour les niveaux en dessous, provoquant une césure assez nette entre les fonctionnaires non mobiles et les dirigeants en emploi fonctionnel. « $L a$ structure vit, fait son boulot, mais dans les moments critiques, la DG est quand même l'instrument de la Présidence, c'est très difficile à vivre, il y a une pression monstrueuse. » DGA Département.

Les séparations conflictuelles avec l'organisation sont difficiles à gérer pour les dirigeants qui sont renvoyés à un certain isolement dès lors que la relation de confiance dont nous parlions plus haut avec le chef de l'exécutif est rompue: «Moi j'ai vu, puisque j'ai vécu deux décharges de fonction, ah oui, j'ai pas eu une carrière facile, euh, et bien, vous êtes très vite un homme seul. Parce que même le personnel avec qui vous vous entendez très bien, vous n'avez aucun problème, dès que il y a rupture de confiance entre le Président ou le Maire, et son premier collaborateur, le personnel passe à autre chose! Mais c'est clair : 'qui est le suivant ?' (...). Donc on est, on est un homme seul. » DGS Département. Nous avons aussi retrouvé trace de cette distanciation ou de lien plus prégnant des fonctionnaires vis à vis de leur collectivité au regard du lien plus distendu face à leur hiérarchie, envers laquelle ils peuvent exprimer loyauté prudente ou attentisme : «Depuis la crise sur le projet que j’ai vécu avec mon DGA, je me dis : je fais mon boulot aussi bien que possible, parce que ça rend service à mes collègues mais il a pas besoin d'attendre de moi plus d'implication. Moi, dans 5 ans, je serais encore là, alors que lui... Vous savez, des DG on en a déjà vu passer, des biens et des moins bien!» Chef de projet Ville moyenne. Sans doute cette césure entre fonctionnaires et équipe de direction renvoie-t-elle à des logiques de fonctionnement assez différentes, mais elle renforce parallèlement la cohésion interne de l'équipe de direction qui travaille à maintenir son unité et à élaborer collectivement les réponses aux attentes politiques (Durat, $2010^{6}$ ) afin de rendre la pression supportable par la responsabilité partagée des choix organisationnels entre le DGS et ses adjoints.

\section{Rétroaction de l'évaluation sur l'activité des dirigeants}

L'évaluation de la performance des dirigeants territoriaux n'est pas nécessairement construite sur des critères clairs et partagés et provoque une anticipation par les directeurs de services des conséquences qu'elle peut avoir sur leur activité voire leur maintien en fonction. «A un moment donné, comme dans l'entreprise, le Directeur Général ou le PDG peu importe, c'est

\footnotetext{
${ }^{6}$ Durat L. Dirigeants de la fonction publique territoriale, une recherche en analyse de l'activité. Savoirs $n^{\circ} 22$. 2010 .
} 
le meilleur quand tout va bien, dès que ça va mal, c'est la bête à tuer, quoi, il est mauvais, etc. » DGS Département. Si la légitimité à exercer son activité est octroyée au départ au DGS par contrat avec l'élu, elle est néanmoins infirmée ou confirmée en cours d'activité par l'évaluation de ses actions. Le dirigeant territorial est ainsi soumis à des 'jugements évaluatifs informels' (Laroche, 2000) par l'autorité territoriale dont il dépend, mais également de jugements issus de nombreuses sources.

Ces sources multiples de jugements évaluatifs informels (exécutif, élus, partenaires institutionnels, voire services eux-mêmes) ont été identifiées à travers les énonciations des dirigeants ; ces évaluations sont relayées par les interventions des DGS en séance de travail collectif, et tracent ce faisant un cadre normé à l'activité dirigeante. Au vu des situations observées, il semble clair que les dirigeants anticipent les stratégies non-explicites du chef de l'exécutif, des élus, voire du cabinet. Ce qui ne manque pas de produire parfois des dilemmes entre la performance reconnue par les élus (du point de vue de la logique politique) et la performance telle que projetée par les dirigeants (du point de vue de la logique administrative) logiques, qui si elles se rejoignent parfois, ne le font ni spontanément, ni sans construction délibérée dans ce sens.

Quelle est la nature de cette évaluation ? Il ne s'agit visiblement pas d'une vision classique de l'évaluation de l'action (qui serait discontinue, fondée sur les résultats, rétrospective et autoréférentielle). Bien plutôt, cette évaluation est multiple (sources diverses du jugement), continue (de nombreux objets de l'activité au cours du mandat sont sensibles, nous l'avons vu plus haut), fondée sur des indices (un élément factuel isolé, une relation, ou un processus peuvent servir de matériau à ce type d'évaluation) et aussi prospective (sur la capacité du dirigeant à proposer des solutions aux élus par exemple). Quant aux références servant de critères à l'évaluation, ils ne sont pas nécessairement le fait d'une seule personne, ni non plus internes (la comparaison à d'autres collectivités, l'indexation de l'organisation sur des référents externes peuvent aussi être pris en compte), nous pouvons donc conclure à une forte instabilité des critères de l'évaluation. Certains DGS rendent compte ainsi des retombées de l'expression de la satisfaction/insatisfaction des citoyens (voire de l'instrumentalisation de celles-ci par d'autres acteurs) : «Pour être clair, quand vous êtes à la tête d'une ville, vous en prenez plein la gueule tous les jours! Regardez le citoyen, comment il fonctionne : vous avez des problèmes avec les gens du voyage, vous avez des problèmes avec les commerçants qui sont jamais contents, vous avez des problèmes avec le plan de circulation, vous avez des problèmes avec les logements sociaux, tout va toujours mal dans une ville! Et puis c'est faux, 
mais c'est quand même, le quotidien c'est quand même ça. Les gens râlent tout le temps ! Le lundi matin, on fait le point de la casse du week-end! » DGS Grande agglomération.

De ce fait, l'anticipation de cette évaluation continue, instable et aux contours larges forme un élément organisateur de toute l'activité du dirigeant en produisant une vigilance aux résultats, aux méthodes employées, à la préparation des actions, et fait produire au DGS et partant, à ses collaborateurs, des principes d'action qu'il édicte, rappelle et reformule à différentes occasions. «- C'est de l'auto-évaluation ? On ne vous donne pas de signal...? - Les bons oui, les mauvais jamais! Mais bien sûr! C'est très rare, on a rarement des collègues qui sont dans cette situation qui sont prévenus que ça se passait pas bien, rarement! Ou ils n'ont pas voulu l'entendre. Donc il faut avoir ses propres indicateurs et ses propres signaux. » DGS grande agglomération.

Ainsi, l'anticipation de l'évaluation telle qu'elle est instrumentalisée par les élus et le cabinet («On est sous l'évaluation du Cabinet constamment.» DGS ville moyenne), génère dans l'activité du dirigeant, un faisceau d'actions de gestion du risque, de prévention, de

préparation, d'influence dans le processus décisionnel, et fait du dirigeant effectivement «l'homme sécuritaire » évoqué par l'un d'eux, mais non pas seulement au titre de la production des actions elles-mêmes, mais bien aussi de la production des conditions de réception de ces actions et donc de poursuite de l'activité dirigeante.

\section{Ressources et stratégies adaptatives déployées par les dirigeants}

Dans cette situation où nous avons pointé les fortes pressions en termes d'atteinte des objectifs, d'évaluation de la performance, de fragilité de la légitimité managériale qui pèse sur les dirigeants territoriaux dont l'action est calée sur le mandat électoral des élus qui le recrutent, il est également un certain nombre de ressources mobilisables par le DGS. Il s'agit en particulier des interactions sphère professionnelle - sphère privée qui représentent à la fois un coût psychologique et des répercussions socioprofessionnelles de la mobilité (pour l'individu et sa famille) mais aussi une ressource pour y faire face. Les équilibres nouveaux sont à réinventer à chaque mobilité, pour bien des dirigeants ils peuvent devenir lourds et les témoignages recueillis sont assez édifiants. Des stratégies défensives ou d'évitement sont alors bâties par les dirigeants territoriaux.

\section{L'articulation vie privée et vie professionnelle}


La question de l'articulation vie privée - vie professionnelle soulève des interrogations en tiroirs et la littérature fait état de conflits, notamment de pressions de rôles venant du travail et de la famille qui seraient dans une certaine mesure mutuellement incompatibles (Kahn et al., $1964^{7}$; Greenhaus et Beutell, 1985 ). Les sources identifiées de ce conflit (Colle \& alii, $2006^{9}$ ) sont le travail atypique, les besoins en mobilité géographique des entreprises et la pénibilité du travail (salariés dont les horaires de travail sont lourds, variables ou imprévisibles, ceux dont le travail exige un niveau d'efforts physiques élevé ou encore ceux connaissant une insécurité de l'emploi). Nous retrouvons en partie ces éléments auprès des dirigeants de la fonction publique territoriale ; les horaires de travail des DGS sont lourds (53 heures hebdomadaires en moyenne mesurées sur 8 mois d'agendas); "Les contraintes sont maximales, je pensais ne pas pouvoir aller au-delà des horaires que j'ai connus au Ministère où je rentrais vers 23 heures, mais ce n'est pas beaucoup plus tôt ici. »; s'il n'est pas question d'efforts physiques, toutefois il est fait référence à une pression intense, présentant une dimension physique et psychique (les verbatim convergent et sont très nombreux, en voici quelques uns : «Elle est permanente, la tension! Elle est permanente, elle est permanente!» DGS grande agglo ; «Il y a une très grosse pression, parce qu'on est charrette sur le plan de mandat»; DGA ville moyenne «La pression est très forte, ce qui parfois inhibe les initiatives individuelles.» DGA grande agglo. «La difficulté, c'est la pression! Une dimension réellement psychologique et physique du poste qui est difficile.» DGS ville moyenne) ; enfin, la faible durabilité dans l'emploi peut être considérée comme une forme d'insécurité.

Les résultats attendus pourraient donc être l'expression d'un fort conflit travail-famille. Dans le cas qui nous occupe, si nous trouvons bien, conformément aux études du domaine, de nombreuses confirmations des difficultés de conciliation de la vie familiale et professionnelle, l'expression forte des satisfactions au travail des DGS et des modes de régulation de difficultés d'articulation sphère privée-professionnelle amène à nuancer les approches classiques du conflit travail-famille.

\footnotetext{
${ }^{7}$ Kahn R.L., Wolfe D.M., Quinn R., Snoek J.D. Et Rosenthal R.A. Organizational Stress, New York: Wiley. (1964).

${ }^{8}$ Greenhaus J.H. Et Beutell N.J. Sources of conflict between work and family roles, Academy of Management Review, vol. 10, p.76-88. (1985).

${ }^{9}$ Colle R., Frimousse S., Peretti J.-M. (2006). Le défi de l'articulation vie privée - vie professionnelle des couples à double carrière, XVIIe Congrès de l'AGRH - Le travail au coeur de la GRH, IAE de Lille et Reims Management School, 16 et 17 novembre 2006.
} 
Les difficultés exprimées par les dirigeants eux-mêmes et leurs conjoints concernent: la professionnalisation (impossibilité d'accéder à certains emplois en raison de la carrière publique du conjoint, difficultés à retrouver un emploi, renoncement à une carrière évolutive), la scolarisation et l'adaptation des enfants, le logement, la vie sociale, les relations amicales :

«Quand on s'est rencontré, j'étais collègue de P., mais c'était impossible, les gens se taisaient quand j'entrais dans un bureau, c'était très dur, j'étais mise à l'index, carrément, les relations étaient difficiles. J'ai dû renoncer à travailler dans la même collectivité»; «A C., bien, j'avais fait mon réseau en termes de travail, relations professionnelles, de modes de garde, d'amis. Il ne s'est pas entendu avec B. (le Maire), il a fallu partir. C'était un traumatisme pour moi. » Epouse DGS ; «Pour moi ça a toujours été difficile d'avoir un mari absent, qui travaille 50 à 60 heures par semaines. Depuis 15 ans de mariage, il y a toujours les deux mêmes points d'achoppement : son temps de travail excessif et le fait de ne pas pouvoir se construire professionnellement dans la durée, dans la stabilité. C’était pas celuilà, mon projet de famille. » Epouse DGA ville moyenne ; «Ce n'est pas facile de reconstruire à chaque fois, c'est très lourd, il faut gérer l'école pour les enfants, leurs activités, le métier de parent. On navigue dans le court terme. On a cette aspiration à s'enraciner quelque part, qui est chaque fois remise en question. On ne peut pas faire les choses dans la durée, c'est douloureux parfois. » Epouse DGS Région.

Face à ces difficultés, les discours recueillis font état de quelques modes de régulation trouvés par les dirigeants vivant en famille : adaptation de l'organisation, compromis, retrait de la part du conjoint ou investissement professionnel comparable :

«J'étais d'abord à B. puis à C. pendant 3 ans, nous avions des réunions très tardives, à partir de 20 h3 et plus. L'organisation de M. me convient mieux, pour la famille, pour voir mes enfants qui sont encore petits.» DGS grande agglomération ; "J'étais d'abord DGS à B., à 32 ans, une ville de 56000 habitants. C'était une pression! J'étais dans une sorte d'exaltation, de réinvention du monde, la passion! J'étais dans ma bulle et j'y étais bien. Là mon épouse a dit stop !! Il n’y a pas de limites, pas de limites; mais je peux lui faire confiance, elle sait me faire prendre en compte les exigences familiales, je m'appuie sur elle pour cela.» DGS grande agglomération; «Ca ne pose pas de problème à ma femme, elle travaille autant que moi et nos enfants sont grands! »DGS Région ; «Disons qu'on a fait un choix d'axer sur son métier plutôt que sur le mien, il y a X années déjà, parce que sinon, ce n'est pas possible.» Epouse DGS Département. 
Quelques auteurs dont les recherches récentes participent à éclairer ces données en examinant les bénéfices potentiels d'un domaine sur l'autre, du travail vers la famille et de la famille vers le travail. Cette perspective bi-directionnelle utilise le concept d'enrichissement travailfamille selon lequel le travail et la famille bénéficient l'un de l'autre, alors qu'ils fournissent chacun des ressources aux individus (habiletés, perspectives, flexibilité, capital social physique et psychologique, et ressources matérielles) qui renforcent l'estime de soi, et peuvent aider les individus à se réaliser dans les autres domaines de la vie. Le lien familial notamment, peut soutenir l'individu, crée une énergie utile pour réaliser des performances dans d'autres rôles. Ce rôle de soutien apparaît clairement dans les entretiens, qu'il concerne les situations délicates ou la vie professionnelle normale :

«J'ai vécu l'époque où on lui a retiré tout, délégation, même la voiture de fonction, il n'avait plus que le téléphone et un bureau vide, il fallait qu'il soit à son travail. Donc le matin, j'emmenais le fils à l'école, le mari au travail. J'ai connu ça. Donc si vous voulez, on est un peu blindé, hein! C'est très dur. (...) Si on est au placard, c'est déjà pas facile, mais il faut déjà pouvoir rebondir, quand il y a des changements politiques, on n'est pas tout seul sur le marché, il y en a combien qui sont sur le carreau ? » Epouse DGS Département ; «Il y a des moments, il faut faire des choix dans la vie, voilà. Il y a eu un fils, je l'ai élevé au début, quand mon mari faisait ses études, il faut savoir que bien des week-ends je les ai passés toute seule avec mon fils, pendant que lui était enfermé dans les bureaux, donc, on n'a rien sans rien.» Epouse DGS Région.

Aucun dirigeant interrogé n'a fait état de difficultés dans sa carrière en raison de ses responsabilités familiales. «Si le travail est ennemi ou rival de la famille, la famille est alliée du travail », selon la formule de Dumas $\left(2006^{10}\right)$ ce qui se vérifie auprès des dirigeants de la fonction publique territoriale interrogés. Ainsi, sur le plan des interactions des sphères professionnelles et privées, ces résultats laissent apparaître par-delà les difficultés de conciliation (et peut-être en raison même de ces difficultés caractéristiques du mode de vie en famille), le soutien apporté par le conjoint et les enfants et son un impact positif sur l'adaptation du cadre dirigeant. Soutien et difficultés des membres de la famille vont de pair, comme le soulignent dans leurs travaux Merignac \& Roger $\left(2005^{11}\right)$.

\footnotetext{
${ }^{10}$ Dumas M. Conflit et enrichissement travail et famille et implication : une étude de relation entre ces construits XVIIe Congrès de l'AGRH - Le travail au coeur de la GRH, IAE de Lille et Reims Management School, 2006.

${ }^{11}$ Merignac O., Roger A. L'impact du conjoint et de la famille sur l'adaptation des cadres expatriés, Revue de Gestion des Ressources Humaines, ${ }^{\circ}{ }^{\circ 58}$. (2005).
} 


\section{Des stratégies d'adaptation souvent défensives}

La flexibilisation accrue de la relation d'emploi a conduit à divers comportements d'anticipation des cadres dirigeants observés, en termes identitaires et professionnels, ce qui a pu faire dire à Perrin: «(la) capacité d'adaptation et d'innovation apparaît comme l'une des vertus majeures des personnels d'encadrement de la fonction publique territoriale » (2005, op cit p.585).

D'une part l'anticipation porte sur la fin de la relation d'emploi : afin de maintenir une image de soi positive et sans doute également pour éviter des conflits concernant les modalités de départ, les directeurs de services sont conduits dans certains cas à garder la main sur la décision de la rupture du détachement. «Faut partir du principe que le politique est un ingrat. Oui, c'est quelqu'un qui a de la bouteille qui peut vous dire ça, il faut toujours anticiper, quand les choses se passent mal, faut les deviner et faut partir avant, toujours » DGS grande agglomération. «Vous savez, il y a un moment, si on sent que ce n'est plus ça, il faut partir. Et le DGS est dans cette situation aussi. Et d'ailleurs pour le DGS plus il est bon, plus il devra partir à un moment donné. - Au bout d'un moment, si le DGS est bon, les élus vont trouver qu'il prend trop de place, qu'il prend le pouvoir, et là, ils voudront le faire partir, c'est inévitable! Alors tant que le Maire tient et le défend, ok, mais il arrive un temps où ça devient délicat pour lui de donner raison au DGS contre ses propres adjoints. Il faut le sentir avant qu'on ne vous le dise. Dans ce cas, il y a une régulation par la mobilité pour le DGS et pour le Dir Cab. » Directeur de Cabinet de grande agglomération.

L'adaptation anticipée est intégrée par Cerdin $\left(1999 ; 2000^{12}\right)$ en tant que variable ayant également une influence sur la mobilité. Le choix des affectations suivantes va effectivement parfois être lié à une volonté de préservation de soi, de retrait afin d'être moins exposé au stress que nous évoquions pus haut (comme l'illustre deux cas recueillis : un DGS qui a pris pendant quelques années une fonction de DGA pour n'être plus en $1^{\text {ère }}$ ligne et un DGS qui est passé d'une direction des services d'une ville à celle d'un département, dans lequel il dit que les conflits fonctionnaires - élus sont moins durs). Ces comportements ont d'autant plus d'importance que l'on se souvient que les membres des équipes de direction auront à gérer leur propre replacement : dans ce contexte, il est préférable d'acquérir et de conserver des ressources sociales réutilisables, et la réputation en fait partie : «Vous savez, c'est un petit

\footnotetext{
${ }^{12}$ Cerdin J-L. La mobilité internationale - Réussir l'expatriation, Paris, Editions d'Organisation (1999).

Cerdin J-L. Recrutement et sélection des expatriés. Gestion 2000, 4, 87-101 (2000).
} 
monde, un tout petit monde. Tôt ou tard tu apprends par la bande les manies de tel politique, les faits d'armes d'untel, les conneries de tel collègue que tu croises ensuite dans une autre réunion. Faut pas pratiquer la politique de la terre brûlée... » DGA Région.

Précisément, l'attention portée à la constitution de son propre capital social nous a semblé être prégnante, presque évidente, particulièrement auprès des directeurs les plus expérimentés. En témoigne le comportement de réseau de nature à faciliter l'évolution professionnelle du dirigeant dans un environnement où les carrières sont de moins en moins linéaires. Ayant des répercussions à la fois individuelles et pouvant être profitables également à son organisation, le réseau de contacts du dirigeant est mobilisé lors d'actions particulières, pour solliciter un conseil, une analyse d'un pair situé hors de sa collectivité territoriale voire même pour recruter des fonctionnaires côtoyés dans un poste préalable, dont le dirigeant a pu évaluer la compétence. «il y avait un gros problème au SDIS, le colonel n'arrivait pas à gérer ses troupes, il y avait un problème de syndicat, enfin c'était compliqué, et donc euh, j'avais joué le rôle de médiateur en recevant le colonel, les syndicats, ensuite tout le monde ensemble etc. et pour ça j'avais appelé le colonel que j'avais connu à A., le patron du SDIS de la région C., avec qui j'avais établi de bonnes relations et je lui avais expliqué le problème dans mon département, je lui ai dit : 'voilà le problème, qu'est-ce que vous en pensez ?'. Les compétences, faut aller les chercher là où elles sont, quoi, hein. » DGS Département ;

Les DGS observés ont pour la plupart fait référence à cette pratique qui consiste à établir des relations avec des personnes qui peuvent potentiellement apporter une aide 'individu pour sa carrière ou son travail, la majorité d'entre eux appartient par exemple à des associations professionnelles. De fait, si tout individu est connecté à des personnes clés dans ou hors l'organisation, le capital social demande à être entretenu et structuré pour atteindre son but ce qui représente un coût et une motivation particulière (Ventolini, 2006). Ce comportement semble d'autant facilité que la nature même des fonctions managériales invite le directeur des services et ses adjoints à être des agents de liaison entre différents partenaires dans et hors de l'organisation, dès lors créer et entretenir des relations fait partie de son rôle.

L'anticipation consiste aussi pour les DG à prévoir eux-mêmes leurs formations, voire leur choix de mobilité, afin de se doter des meilleurs atouts pour le parcours à venir: ainsi d'acquérir les compétences dont ils font le constat qu'elles sont manquantes et fondamentales pour leur évolution de carrière, voire de prendre des postes leur permettant de se confronter à des situations formatrices. Certaines carrières peuvent être accélérées par une mobilité bien organisée, géographique mais surtout d'une spécialité ou d'un niveau d'administration à un 
autre, comme le remarque Perrin (2005, op cit; p. 591) : «une période d'apprentissage consacré aux ressources humaines, aux questions juridiques, aux marchés publics permet de se constituer une carte de visite que les employeurs ne manqueront pas d'apprécier».

Ainsi avons-nous pu observer ce comportement à l'œuvre chez 5 directeurs, l'un pour se former aux ressources humaines, deux autres pour compléter leur compréhension de la gestion financière, un dirigeant prenant un poste d'expert sur le développement durable, le dernier choisissant son affectation pour pouvoir se former à la mutualisation des services, dans l'objectif d'en faire une valeur ajoutée pour leurs prochains postes.

Un dernier effet de la mobilité est repéré : la transformation du contrat psychologique qui lie l'organisation au dirigeant, donnant à voir un affaiblissement de la relation de confiance qui unissait historiquement les cadres à leur entreprise (Bouffartigue, 2000 ${ }^{13}$ ) et un moindre sentiment d'appartenance, comme ça nous a été formulé par un DGS : "C'est ce qui me séduit le plus, l'indépendance : je peux me tirer quand je veux. Ça ne me gêne pas que l'on me considère comme un mercenaire, pourquoi pas ? Je suis l'homme du moment, il n'y a pas d'amitié possible avec les élus, pas de proximité. Ils vous font en permanence sentir leur légitimité, la légitimité démocratique. Je ne me fais pas d'illusion, j’ai vu la déception de collègues DGS antérieurement. Ça illustre bien la phrase d'André Fontaine : ' ils dorment dans le même lit, mais ils ne font pas le même rêve' »DGS Région. Comme on le perçoit, cette posture n'est pas dénuée d'amertume et peut-être de renoncement à un idéal préalable, mais elle est le reflet d'une rationalisation en réponse à l'insécurité créée par la succession de mobilités, tendant à prendre acte de la relation contractuelle et individualisée à l'employeur.

\section{Discussion et conclusion}

L'étude révèle la spécificité de l'objet d'étude et sa complexité. Si la recomposition managériale est une condition d'exercice récente dans la fonction publique territoriale, calant la durée de l'activité du dirigeant sur le mandat électoral de l'élu, elle est identifiée par les principaux intéressés comme faisant partie intégrante de la gestion territoriale. Cette temporalité nouvelle renforce l'implication des dirigeants auprès de l'autorité territoriale qui les a recrutés mais produit des relations ambigües aux élus et rétroagit sur les modalités de l'activité conduite. L'évaluation multiforme de l'action du directeur des services produit un travail incessant de construction de sa légitimité (non acquise d'emblée ni durablement) par le dirigeant. De fait, si le risque de sanction est plus présent, la rupture du détachement signifie

\footnotetext{
${ }^{13}$ Bouffartigue p., Gadea C., Sociologie des cadres, Paris, Editions La Découverte (2000).
} 
également une reconnaissance de l'influence du dirigeant sur un traitement des dossiers qui n'est plus purement technique mais politico-administratif au niveau de l'équipe de direction managériale, les élus souhaitant recruter une équipe à leur convenance. La question se pose alors de l'intérêt organisationnel des recompositions managériales à chaque alternance : il apparaît que la relation de confiance avec le recruteur et employeur en la personne du chef de l'exécutif de la collectivité territoriale soit la première garantie de fonctionnement pour le dirigeant, cette proximité conduisant tout à la fois à cliver les membres de l'équipe dirigeante nommés sur emploi fonctionnel (càd susceptibles d'être mobiles) plus proche de l'équipe politique des autres fonctionnaires.

Complexité des résultats obtenus, ensuite : le système de carrière se voit remplacé par une logique de l'emploi individualisé qui produit des effets profonds ; fragilisation de leur position, mais intérêt individuel en termes matériels et en termes d'apprentissage ; sur le plan de l'équilibre vie professionnelle vs extraprofessionnelle, si on repère bien un conflit travailfamille marqué, celui-ci s'accompagne de modes de régulation et ne semble en rien nuire à la carrière des dirigeants, bien au contraire la notion d'enrichissement des deux domaines s'applique tout à fait, la famille jouant pleinement le soutien au dirigeant ; la responsabilisation quant à la gestion de la carrière semble également accrue (anticipation de la fin de la relation d'emploi, prise en compte de la formation, choix des mobilités, comportement de réseau) bien que l'on puisse s'interroger sur cette intériorisation de processus qui gagnerait peut-être à être pris en compte collectivement.

Les tendances déjà présentes de recouvrement de la sphère administrative et politique sont renforcées par la fonctionnalité de l'emploi de DGS et si ce mouvement institutionnel ne bouleverse pas en profondeur les rapports existants entre les acteurs il donne néanmoins le jour à un nouveau mode d'exercice du métier de dirigeant territorial. Face à de plus hauts niveaux d'insécurité (relations d'emploi de plus courtes durées, stress lié aux divers facteurs politiques) les membres des équipes managériales ont une double réponse : d'une part un surinvestissement et d'autre part l'élaboration de stratégies défensives très concrètes.

Ainsi il ressort que la perception d'insécurité est centrale dans la production d'effets tant individuels qu'organisationnels de la fin de détachement sur emploi fonctionnel. Cette insécurité conduit probablement à des effets positifs (réajustements cognitifs, engagement et 
processus identitaires selon Beauvois \& Joule, $1981^{14}$ ). Mais l'insécurité présente le risque de se transformer en spirale régressive pour l'individu si elle dépasse un certain seuil, or comment évaluer celui-ci ? Combien de dirigeants éprouvent une usure professionnelle accélérée face à de telles contraintes, et dès lors, quel est le coût humain et la durabilité d'une performance liée à l'insécurité ? De plus les effets organisationnels produits sont-ils garants de performance ou d'apprentissage organisationnels ? La question peut se poser au regard des précautions prises pour l'évitement des conflits, la recherche de consensus et de légitimité constante : sont-ce des garanties de cohésion ou du temps et de l'énergie qui auraient pu être investis dans la production de services?

Les enseignements de cette recherche sont d'ordre théorique et opérationnel, permettant une connaissance plus fine à la fois de l'activité des dirigeants de la fonction publique territoriale, et une approche approfondie de l'emploi fonctionnel d'autant plus intéressant à observer que le phénomène date d'une quinzaine d'années dans la fonction publique territoriale. En matière de gestion de ressources humaines, quelques pistes pourraient être suivies par les instituts de formation et le CNFPT: une attention à la gestion de l'accès aux responsabilités, aux transitions entre deux postes, et à la sortie de ce type de carrière qui gagneraient en fluidité à ne pas reposer sur les seuls individus, mêmes dotés de réseaux efficaces. Des propositions de formation ou des fonctions d'audit, d'expertise, ou encore des missions ponctuelles pourraient être imaginées durant ces périodes pour permettre à la fois la distanciation et la réflexivité, voire le partage d'expériences et l'analyse des pratiques pour les dirigeants concernés. Aujourd'hui, la dissociation de la gestion des ressources humaines ne le permet guère, ce qui rend la transversalité plus délicate. Enfin, la préparation des administrateurs au sein des instituts de formation de la fonction publique territoriale gagnerait sans doute à intégrer la dimension de gestion de la mobilité. Ainsi la carrière pourrait-elle être conçue comme un véritable dispositif de formation, faisant jouer un rôle majeur aux parcours professionnels (enchaînement de postes, de missions, de projets pour structurer l'apprentissage par l'expérience) dans le développement des futurs dirigeants.

\footnotetext{
${ }^{14}$ Beauvois J.L., Joule R. Soumission et idéologies. Paris, PUF. (1981).
} 


\section{Annexe méthodologique}

La méthodologie qualitative utilisée est fondée sur ce qu'on appelle en psychologie du travail et ergonomie une analyse du travail en vue de la formation (Leplat 1974) pour saisir l'activité collective des dirigeants telle qu'elle s'exerce réellement, et non telle qu'elle est prescrite. Cette analyse de l'activité des dirigeants de la fonction publique territoriale a été menée à la demande de l'Institut d'Etudes Territoriales (INET) qui forme ces futurs dirigeants. L'échantillon déterminé pour l'enquête est composé de 12 collectivités territoriales (CT) : 2 régions et 1 département de plus de 900000 habitants, 1 département de moins de 900000 habitants, 3 grandes communautés d'agglomérations et 2 grandes communes (plus de 150000 habitants) et 3 communes moyennes (entre 35000 et 65000 habitants). Un ensemble de 25 dirigeants membres de Direction Générale (Directeurs généraux des services, Directeurs Généraux adjoints, que nous désignerons DGS et DGA), cœur de cible de la recherche, et 23 partenaires de leur activité (élus, cabinet, chargés de mission, secrétaire et conjoints quand cela a été possible) ont fait l'objet de l'enquête, et ont été rencontrés sur leur lieu d'exercice de l'activité.

Pour l'ensemble du recueil discursif (66 entretiens semi-directifs enregistrés, d'une durée de $1 \mathrm{~h} 15$ à 2h45), les investigations de l'étude ont porté sur 48 interlocuteurs intégrées dans un dispositif de 28 journées d'observation de l'activité à réalisées in situ, ainsi que le recueil de traces. L'observation, le recueil de traces (CV, fiches de postes, organigrammes) et de discours (entretiens semi-directifs et entretiens de retour sur activité) s'est déroulé dans la plupart des cas pendant une semaine et au moins deux journées. L'observation participante a été menée par 'filature de l'acteur' en recherchant sur chaque terrain : le traitement des informations entrantes, le type d'interactions entre acteurs, le type d'intervention du dirigeant, dans son activité propre, dans les modalités d'organisation de son agenda, dans les réunions de l'équipe de direction et dans les entretiens bilatéraux avec ses collaborateurs. Elle a été complétée de prises de vues sur l'environnement de travail, repérages des espaces (plans de bâtiments et affectation des lieux/position des acteurs) et de la circulation des acteurs dans ces espaces. 